Mamchur A. A.

\title{
POSSIBILITIES FOR INVOLVEMENT OF TRANSFORMED MULTIPOTENT MESENCHYMAL STEM CELLS IN SARCOMOGENESIS AS TUMOR-INITIATING CELLS
}

\section{RESUME}

In recent years the concept of tumor stem cells or tumor-initiating cells has gained a wide recognition. Hence the amount of data pertaining to involvement of the latter in the sarcomogenesis increases. At the time being the properties of the tumor-initiating cells are actively studied. As has been found, they are characterized by self-renewal, high migration potential and high level of resistance to chemotherapy. The given characteristics are also analogous for the multipotent mesenchymal stem cells (MMSCs) which are involved in the regeneration processes. The above-said can point to the possibility of the existence of the transformed MMSCs analogs as tumor-initiating cells. In turn, the transformed MMSCs can differ by immortal phenomenon conditioned by high level of telomerase expression, antigens set, genetic and genomic changes. As a consequence, the transformed MMSCs attain potential to form sarcoma.

KEYWORDS: multipotent mesenchymal stem cells; tumor-initiating cells; sarcomogenesis

The multipotent mesenchymal stem cells (MMSCs) play an important role in the regeneration processes. It is known that the MMSCs were first obtained by Fridenstein as fraction of the bone marrow cells which were characterized by adhesion to plastic surface and colony-formation during their ex vivo culture [1,2]. It is noteworthy that the MMSCs were discovered from other tissues like subcutaneous adipose tissue, muscles, umbilical blood and others.

Unfortunately there are no antigen determinants characterizing MMSCs. Nevertheless these cells are characterized by many researchers as positive for $C D 73, C D 190$ and $C D 105, C D 44$ and negative for $C D 45$, CD34 and CD31. Besides, the MMSCs posses targeted differentiation in the osteogenic, adipogenic, chondrogenic and myogenic directions [3-8]. Apart from this, bone marrow and subcutaneous adipose tissue MMSCs are easily obtained and ex vivo cultured.

The regenerative and immune suppressive properties of MMSCs have led to numerous clinical trials exploring their utility in the regenerative medicine and tissue engineering $[9,10]$. In parallel, investigations are currently being conducted on their use in the treatment of a variety of diseases (e.g., burns, bone fractures and degenerative injuries of the extremities). For their use in clinic the necessary amount of MMSCs is to be cultured ex vivo. The cultured cell material is subsequently frozen for saving. The above procedures may frequently lead to genetic changes and transformation of MMSCs. Hence there arises a problem of possible danger of using MMSCs in clinical practice from the viewpoint of oncology.
Moreover, recent investigations have unfortunately found that both human and animal MMSCs are able, under certain conditions, to take part in the formation and progression of tumors. In the first case they can stimulate tumor growth and metastasing as a component of both, tumor micro environment [11-17] and tumor initiating cells [18-21]. At the same time, possible stimulation of tumor growth and their metastasing by mesenchymal stem cells is disputable as several investigators point to their opposite effect [22-24]. In the second case transformed MMSCs act like cancer stem cells or tumor-initiating cells. Alongside there are opinions that human mesenchymal stem cells are unable for spontaneous transformation during their long-term ex vivo culturing [24, 25] that makes them differ from MMSCs of mice and rats. However some investigators point to the significant possibility of spontaneous transformation of MMSCs during their long-term culturing [26, 27]. These authors have shown relative safety of subcutaneous adipose cells MMSCs culturing during 6-8 weeks and considerably of their transformation during 4-month culturing (50\% of cells overcome critical phase and continue proliferating). The transformed MMSCs undergo certain changes. For instance, the transformed MMSCs can form micro spheres during their growing in the agarose gel. Many cultures of the transformed MMSCs can grow in the absence of adhesion to surface. Furthermore, the transformed MMSCs possess greater proliferative potential and higher level of telomerase expression.

The transformed MMSCs have certain differences from their normal analogs in the antigen determinants as well. The transformed MMSCs 
have greater expression of CD44, CD166 markers, whereas CD105 is less expressed. Separate antigen determinants characteristic of normal human MMSCs, namely CD73, CD90 and Stro-1 are not detected. Several investigators demonstrated their greatly limited potential to separate kinds of differentiation. After transformation one observes a significant reduction of differentiation in the adipogenic and chondrogenic direction. Instead, the transformed MMSCs have greater potential to osteogenic differentiation under definite conditions. The main property of the transformed stem cells is their tumorogenicity. For instance, G. Rosland and colleagues assessed the tumorogenic activity of spontaneously transformed human MMSCs in vivo by injecting these cells to immunedeficient NOD/SCID mice [27]. Earlier these investigators had conducted eGFP gene transduction for further monitoring of tumor growing in the lungs. On day 9 they registered first foci of tumor growth in the lungs.

Respectively, the animals injected normal MMSCs did not manifest tumor process. The histological analysis of tumors showed that lung metastases were epithelioid by their morphological signs but they had the markers of both mesenchymal and epithelial direction of differentiation. Analogous investigation was carried out by the Rubio group although they used the spontaneously transformed MMSCs of the subcutaneous adipose tissue obtained after long-term culturing. After injection of the given cells to immune-deficient mice the multiple foci of tumor growth appeared in the lung, liver, brain, kidneys and heart [26]. The abovementioned investigations have proved the fact that transformed MMSCs can initiate sarcomogenesis and thus play role of tumor-initiating cells.

\section{THEORY OF CANCER STEMI CELLS}

In view of the above-said it is worthwhile to consider the theory of cancer stem or tumor-initiating cells. First of all, the terms "tumor stem cells" and "tumor-initiating cells" are synonymous. At the same time the term "tumor stem cells" is more known in the literature although it does not adequately reflect tissue genesis of malignancies and it is rather used by tradition.

According to the theory of cancer stem cells or tumor-initiating cells, tumors are formed from stem cells (stem cells from adult organism included) or their progenitors with a high proliferative potential resultant from appearance in the latter of changes in the genetic programs regulating normal tissue regeneration. Besides, changes occur in the micro environment of the transformed stem cell. As a result of such initiation of tumors, there takes place formation of a peculiar hierarchic structure which is resembles regeneration processes of the normal tissues. It is the hierarchy of cells in the normal tissue and malignant tumors with the stem cells at the top forming the pool of progenitors with lesser proliferative and differential potential. The progenitors, in turn, form terminal link of this hierarchic structure - the differentiated cells. The mentioning here should be made of the clonal evolution concept that has dominated in oncology for a long time and determined similar potention for tumorogenesis of all tumor cells. The concept of tumor-initiating cells states that only transformed stem cells can generate tumor, following from their properties for renewal and greater proliferative potential [28-31].

Notably, many sarcomas of various localization are characterized by their resistance to cytotoxic agents thus showing certain analogy with normal stem cells. The greatest level of resistance is characteristic of low-differentiated sarcomas. It is known that MMSCs hyperexpress proteins which are able to eliminate cytotoxic substances [32, 33, 34]. For instance, some investigators provide evidence for P-glycoprotein expression in the latter and reduced level of its expression during cells differentiation [35]. Obviously, the above-mentioned properties are not lost after MMSCs transformation and condition, in turn, the presence of recurrences following chemotherapy. In addition, there is certain analogy between normal stem cell heterogeneity and tumor-initiating cells by their proliferative potential and morphologic properties. The stem cells are characterized by asymmetric division following which there appear de novo stem cells and more differentiated progenitors with a considerably lower proliferative potential and other morphologic characteristics. Analogous processes take place in the tumor but with aberrant differentiation. In accord with the above-said, many investigators have reported the existence in the heterogenic tumor population of a minor/n insignificant fraction of cells capable to generate tumor-initiating cells. This conception has been confirmed many times experimentally. Thus, after transplantation of different cell populations derived from various tumor cells in animals it was seen that only one of them was tumorogenic [28-31].

\section{MODELS OF SARCOMOGENESIS CONDITIONED BY TRANSFORMED MIMISCs}

The two main models of sarcomogenesis induced by transformed MMSCs are being analyzed within the framework of the conception about the existence of tumor-initiating cells. The first model is based on the possibility of MMSCs transformation at different levels of their differentiation. Thus, the given model explains the existence of sarcomas of different level of differentiation. According this model, low-differentiated sarcomas are formed from MMSCs and their early progenitors. Respectively, highly-differentiated sarcomas are formed as a result of oncogenic mutations in terminally differentiated MMSCs. According to this model, phenotypic characteristics, histological structure of the sarcoma are determined depending on the direction of MMSCs differentiation, stage of MMSCs differentiation on which it was initiated [36]. For instance, using this model it is possible to explain different levels of osteosarcoma differentiation [36, 37]. Also, this model has been confirmed by the profile of expression of the genes committed to one or another direction of MMSCs differentiation. For instance, chondrosarcoma cells manifest significant phenotypic affinity to mature chondrocytes. At the same time, the profile of genetic expression of less differentiated form of sarcoma manifests greater analogy with the profile of genetic expression of pre-chondrogenic stage of MMSCs differentiation [36, 38].

As a result of stable constitutive proliferation of MMSCs, a pool of malignant cells with various morphological properties is formed, in which in turn additional mutations occur. Indeed, this non-uniformity is explained by genetic changes in the latter. Further there appears a probability of formation of immortal clone with partial or complete loss of the ability for differentiation that explains formation of sarcomas with various stage of differentiation [36].

There exists another model of sarcomogenesis conditioned by MMSCs transformation. According to this model, due to mutations taking place in MMSCs the latter gain the capacity for constitutional active symmetric and asymmetric proliferation. As a result of constitutional proliferation of MMSCs there forms a pool of the tumor cells with various morphological properties in which in turn additional mutations occur. Such irregularities are explained by genetic changes in the latter. Further there appears the possibility of formation of immortalized clone with partial or complete loss of the ability for differentiation and thereby the formation of sarcomas with varying degree of differentiation is explained [36]

\section{POSSIBLE GENETIC MIMISCS CHANGES INITIATING SARCOMIOGENESIS}

In the context of the above-given conceptions about sarcomogenesis one of its triggers can be the hyper activating mutation of TERT gene coding telomerase. As a result, MMSCs can acquire immortal phenotype with an unlimited proliferating potential. It is known that high endogenous telomerase activity is observed in $70-90 \%$ of malignant cell lines whereas in normal MMSCs the activity one observes more significant activity of this factor. Owing to high telomerase activity, MMSCs are cable to pass the period of crisis during culturing. Unlimited proliferating activity can be conditioned by hyperexpression of $c$-myc oncogene. It is known, that $30 \%$ of MMSCs, which pass aging stage, are characterized with an increased $c-m y c$ oncogene expression. Further increase of this gene expression is seen in the transformed MMSCs. In its turn, $c-m y c$ 
oncogene hyperexpression can be conditioned by inactivating mutations in the genes of inhibitors of cycline-dependent kinases of INK4 family/ group (15INK4B, p16INK4A, p18INK4C and p19INK4D proteins) which, as a rule, can bind with cycline-D-dependent $C d k 4$ and $C d k 6$ kinases $[39,27]$. It is known, that in the absence of inhibiting action of cyclinedependent kinases the latter can phosphorilate $R b$ factor. In the case of $\mathrm{Rb}$ factor phosphorilation there occurs its dissociation with transcriptional E2F1 factor and the latter gains the capacity to bind with c-myc oncogene promoter, thereby stimulating cell proliferation. An unlimited proliferating activity creates predisposition for further accumulation of oncogenic mutations. Under conditions of an increased proliferation the reparation processes are worsened. Therefore, the level of genetic instability can be increased [39]. With increasing genetic instability level the proliferation and differentiation processes are disturbed and lead to the appearance of tumorogenic clone.

As a result of genetic instability of the transformed MMSCs there can be various chromosome aberrations which condition deregulation of the processes of their normal differentiation and proliferation. The so-called fusion genes (genes-chimeras), being formed as a result of chromosomal translocations, play great role in the sarcomogenesis processes. The presence of certain chimera genes serves/ is used as diagnostic marker of various sarcomas. It is known, that Ewing sarcoma possesses EWS-FLI-1 chimera-gene expression. The given chimera-gene is considered as one of the diagnostic markers of the Ewing sarcoma. However, in transduction of human MMSCs by EWSFLl-1 gene one does not observe tumorogenesis in the immunedeficient animals following injection of the given cells. At the same time transduced MMSCs manifested great analogy in the pattern of genetic expression with the pattern of genetic expression of the Ewing sarcoma cells. Obviously, in order for the transduced MMSCs to gain the capacity for tumorogenesis, additional genetic changes are necessary. Analogous investigations were carried out with the use of transduction of chimera FUS-CHOP gene, the presence of which is characteristic for human fibrosarcoma cells. Although injection of transduced MMSCs of the given chimeric gene did not promote tumor formation [40]. The investigators assume that additional combination of mutations is necessary for FUSCHOP- transduced MMSCs to gain their tumorogenic potential. There is an assumption that such mutation can be inactivating $p 53$ gene mutation or its deletion. The above-said investigations indicate that expression of such chimeric genes in MMSCs is obligatory but not sufficient condition in tumorogenesis. The role of given genetic disturbances in sarcomogenesis needs further investigation [41].

\section{CONCLUSION}

IN SUMMING UP, UNDER CERTAIN CIRCUMSTANCES TRANSFORMED MMSCS CAN ACT AS CANCER STEM CELLS OR TUMOR-INITIATING CELLS. THESE FINDINGS WERE ALSO PROVED BY EXPERIMENTAL INVESTIGATIONS IN WHICH TUMOROGENIC POTENTIAL OF THE LATTER FOLLOWING SPONTANEOUS TRANSFORMATION UNDER CONDITIONS OF PROLONGED CULTURING WAS DISCLOSED. OBVIOUSLY THIS PROCESS IS COMPLICATED ENOUGH AND REQUIRES NOT ONLY DEFINITE COMBINATION OF ALTERATIONS IN THE GENETIC APPARATUS OF MMSCS, BUT ALSO DEFINITE ALTERATIONS IN THEIR MICRO ENVIRONMENT. INFLUENCE OF MICRO ENVIRONMENT ON THE PROCESS OF SARCOMOGENESIS IS ALSO IMPORTANT IN THE CONTEXT OF FORMATION OF VARIOUS KINDS OF SARCOMA. IT IS KNOWN, THAT MICROENVIRONMENT OF THE STEM CELLS, INCLUDING MMSCS, CAN COMMIT THEM TO DIFFERENT DIRECTIONS OF DIFFERENTIATION. ALSO, IN THE CONTEXT OF TUMOR-INITIATING CELLS THE PROBLEM OF SAFETY IN USING MMSCS IN CLINICAL THERAPY IS VERY TOPICAL IN VIEW OF THEIR POSSIBLE TRANSFORMATION DURING CULTIVATION FOR FURTHER THERAPEUTIC UTILITY.

1. Augello A, Kurth TB, Bari C. Mesenchymal stem cells: a perspective from in vitro cultures to in vivo migration and niches. European Cells and Materials. 2010; 20:121-133.

2. Zhao-Jun Liu, Ying Zhuge, Omaida C. Velazquez Trafficking and Differentiation of Mesenchymal Stem Cells. J CellBiochem. 2009; 106(6):984-991.

3. Hass $R$, Kasper $C$, Böhm $S$, Jacobs $R$, et al. Different populations and sources of human mesenchymal stem cells (MSC): A comparison of adult and neonatal tissue-derived MSC. Cell Communication and Signaling. 2011; 9(12):1-14

4. Sarugaser R, Hanoun L, Keating A, et. al. Human Mesenchymal Stem Cells Self-Renew and Differentiate According to a Deterministic Hierarchy. PlosOne. 2009; 4(8):e6498.

5. Peister A, Mellad JA, Larson BL, et al. Adult stem cells from bone marrow (MSCs) isolated from different strains of inbred mice vary in surface epitopes, rates of proliferation, and differentiation potential. Blood. 2004; 103(5):1662-1668.

6. Morikawa S, Mabuchi $Y, K u b o t a ~ Y$, et al. Prospective identification, isolation, and systemic transplantation of multipotent mesenchymal stem cell sinmurine bone marrow. J ExpMed. 2009; 206(11):2483-2496.

7. Nadri S, Soieimani M, HosSeni RH, et al. Anefficient method for isolation of murine bone marrow mesenchymal stem cells. Int. J. Dev. Biol. 2007; 51: 723-729.

8. Eslaminejad MB, Nasarian H, Taghiyar L. Mesenchymal Stem Cell Isolation from the Removed Medium Rat's Bone Marrow Primary Culture and their Differentiation into Skeletal Cell Lineages. Yakhteh Medical Journa. 2008; 10(1):65-72.

9. Tae SK, Lee SH, Park JS, et al. Mesenchymal stem cells for tissue engineering and regenerative medicine. BiomedMater. 2006; 1(2):63-71.

10. Caplan A. I. Adult mesenchymal stem cells for tissue engineering versus regenerative medicine. J CellPhysiol. 2007; 213(2):341-347.

11. Mishra PJ, Humeniuk R, Medina DI, et al. Carcinoma-Associated Fibroblast-Like Differentiation of Human Mesenchymal Stem Cells. CancerRes. 2008; 68(11):4331-4339.

12. Mishra PJ, Glod JW, et al. Mesenchymal stem cells: flip side of the coin. CancerRes. 2009; 69(4):1255-1258.

13. Bergfeld SA, DeClerck YA. Bone marrow-derived mesenchymal stem cells and the tumor microenvironment. CancerMetastasisRev. $2010 ; 29(2): 249-261$.

14. Houthuijzen J M, Daenen LGM, Roodhart JML, et al. The role of mesenchymal stem cells in anti-cancer drug resistance and tumour progression. Br J Cancer. 2012; 106(12):1901-1906.

15. Wong R. S. Y. Mesenchymal Stem Cells: Angels or Demons? Journal of Biomedicine and Biotechnology. 2011; 2011:1-8. 
16. Yen BL, Yen ML. Mesenchymal Stem Cells and Cancer — for Better or for Worse? J. Cancer Mol. 2008; 4(1):5-9.

17. Liu $Y$, Han Z P, Zang SS, et al. Effects of Inflammatory Factors on Mesenchymal Stem Cells and Their Role in the Promotion of Tumor Angiogenesis in colon Cancer. J Biol Chem. 2011; 286(28):25007-25015.

18. Han Z, Jing $Y$, Zhang $S$, et al. The role of immunosuppression of mesenchymal stem cells in tissue repair and tumor growth. CellBiosci. 2012; 2(1):2-8.

19. Djouad F, Plence C, Bony C, et al. Immunosuppressive effect of mesenchymal stem cells favors tumor growth in allogeneic animals. Blood. 2003; 102(10): 3837-3844.

20. DeMiguel MP, Fuentes-Julian S, Blazquez-Martinez A, et al. Immunosuppressive properties of mesenchymal stem cells: advances and applications. Curr Mol Med. 2012; 12(5): 574-591.

21. LeBlanc K, Ringdén O. Immunomodulation by mesenchymal stem cells and clinical experience. J InternMed. 2007; 262(5):509-525.

22. Nakamizo A. Human bone marrow-derived mesenchymal stem cells in the treatment of gliomas. Cancer Res. 2005; 65(8):3307-3318.

23. Lu Y R, Yuan $Y$, Wang $X \mathrm{~J}$, et al. The growth inhibitory effect of mesenchymal stem cells on tumor cells in vitro and in vivo. Cancer Biol Ther. 2008; 7(2):245-251.

24. Khakoo AY, Pati S, Anderson SA, et al. Human mesenchymal stem cells exert potent antitumorigenic effects in a model of Kaposi's sarcoma. J ExpMed. 2006; 203(15):1235-1247.

25. Serakinci N, Guldberg P, Burns JS, et al. Adult human mesenchymal stem cell as target for neoplastic transformation. Oncogene. 2004; 23(29):5095-5098.

26. Rubio D, Garcia-Castro J, Martín M C. Spontaneous Human Adult Stem Cell Transformation. Cancer Res. 2005; 65(8):3035-3039.

27. Røsland GV, Svendsen A, Torsvik A, et al. Long-term cultures of bone marrow-derived human mesenchymal stem cells frequently undergo spontaneous malignant transformation. Cancer Res. 2009; 69(13):5331-5339.

28. Zhou $B-B$ S, Zhang $H$, Damelin M, et al. Tumour-initiating cells: challenges and opportunities for anticancer drug discovery. NatureReviewsDrugDiscovery. 2009; 86:806-823.

29. Guo W, Lasky J L, Wu H, et al. Cancer stem cells. PediatrRes. 2006; 59(4):59-64.

30. Yi S, Nan KJ. Tumour-initiating stem cells in liver. Cancer Biol. Ther. 2008; 7(3):325-330.

31. Schatton T, Frank, N Y, Frank M H. Identification and targeting of cancer stem cells. Bio essays. 2009; 31(10):1038-1049.

32. Adhikari AS, Agarwal N, Wood BM, et al. CD117 and Stro-1identify osteosarcoma tumor-initiating cells associated with metastasis and drug resistance. Cancer Res. 2010; 70(11): 4602-4612.

33. Fujii H, Honoki K, Tsujiuchi T, et al. Sphere-forming stem like cell populations with drug resistance in human sarcoma cell lines. Int J Oncol. 2009; 34(5): $1381-1386$

34. Rodriguez R, Rubio R, Menendez P. Modeling sarcomagenesis using multipotent mesenchymal stem cells. Cell Res. 2012; 22(1):62-77.

35. Kim SG, Jeon CH, Suh HS, et al. P-glycoprotein expression in extracellular matrix formation of chondrogenic differentiation of human adult stem cells. Cell Biol Int. 2007; 31(9):1042-148.

36. Mohseny $A B$, Hogendoorn PC. Concise review: mesenchymal tumors: when stem cells go mad. Stem Cells. 2011; 29(3):397-403.

37. Tang N, Song WX, Luo J, et al. Osteosarcoma development and stem cell differentiation. Clin Orthop Relat Res. 2008; 466(9):2114-2130.

38. Boeuf $S$, Kunz P, Hennig $T$, et al. A chondrogenic gene expression signature in mesenchymal stem cells is a classifier of conventional central chondrosarcoma. J Pathol. 2008; 216(2):158-166.

39. Kamilova TA, Novik AA, Tsygan VN. Vvedenie v molekuliarnuiu biologiiu kantserogeneza [Introduction to the molecular biology of carcinogenesis]. GoetarMed. M., 2004. 222 p.

40. Funes J.M. Transformation of human mesenchymal stem cells increases their dependency on oxidative phosphorylation for energy production. Proc Natl Acad Sci U S A. 2007; 104(15): 6223-6228.

41. Rodriguez R, Rubio R, Menendez P. Modeling sarcomagenesis using multipotent mesenchymal stem cells. Cell Res. 2012; 22(1):62-77.

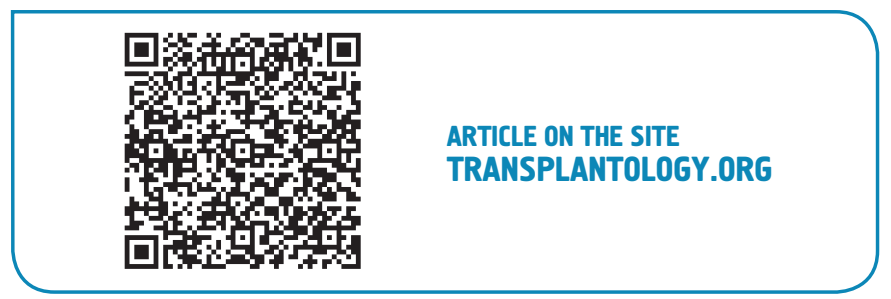

The author indicates no potential conflicts of interest. 